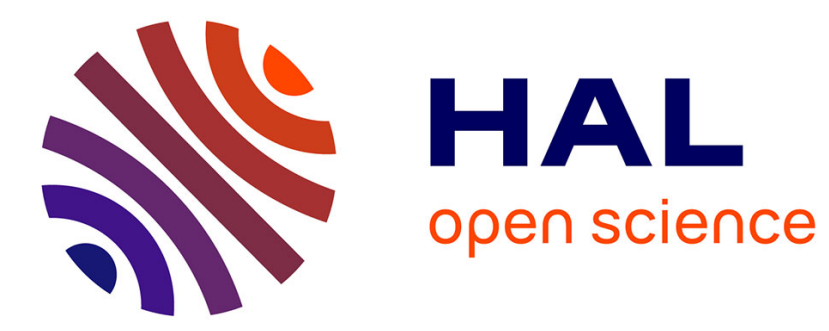

\title{
La stratégie communicationnelle du musée de l'Art brut : considérations d'un récepteur avisé \\ Esther González-Martínez
}

\section{To cite this version:}

Esther González-Martínez. La stratégie communicationnelle du musée de l'Art brut: considérations d'un récepteur avisé. Publics et musées, 1996, 9 (1), pp.105 - 127. 10.3406/pumus.1996.1073 . hal01859234

\section{HAL Id: hal-01859234 \\ https://hal.science/hal-01859234}

Submitted on 21 Aug 2018

HAL is a multi-disciplinary open access archive for the deposit and dissemination of scientific research documents, whether they are published or not. The documents may come from teaching and research institutions in France or abroad, or from public or private research centers.
L'archive ouverte pluridisciplinaire $\mathbf{H A L}$, est destinée au dépôt et à la diffusion de documents scientifiques de niveau recherche, publiés ou non, émanant des établissements d'enseignement et de recherche français ou étrangers, des laboratoires publics ou privés. 


\section{La stratégie communicationnelle du musée de l'Art brut :} considérations d'un récepteur avisé

In: Publics et Musées. N9, 1996. pp. 105-127.

Citer ce document / Cite this document :

Gonzalez Martínez Esther. La stratégie communicationnelle du musée de l'Art brut : considérations d'un récepteur avisé. In: Publics et Musées. N9, 1996. pp. 105-127.

doi : 10.3406/pumus.1996.1073

http://www.persee.fr/web/revues/home/prescript/article/pumus_1164-5385_1996_num_9_1_1073 


\section{Résumé}

L'article présente une analyse de l'exposition permanente du musée de l'Art brut de Lausanne. L'exposition est vue comme un artefact communicatif capable de prendre de multiples significations en fonction des réceptions dont il fait l'objet. Néanmoins, cet artefact, pour être constitué et compris, formule des directives à suivre. L'auteur, devenu visiteur averti, tente de dégager la structure de l'exposition par l'interprétation de données faisant référence autant à sa production qu'à sa réception. II met ainsi en évidence cinq opérations orientatives de la réception considérées comme autant de moments d'une stratégie communicationnelle susceptible d'être à l'origine des réceptions observées.

\section{Resumen}

Este articulo presenta un analisis de la exposición permanente del Museo de Arte Bruto (musée de l'Art Brut) de Lausana, Suiza. La exposición es considerada como un artefacto comunicativo capaz de adoptar distintos significados segùn las recepciones de las cuales es objeto. Este artefacto incluye instrucciones para ser recreado por el publico. La autora saca a la luz la estrutura de la exposiciôn mediante la interprétation de datos que hacen referencia tanto a la production como a la recepciôn del museo. Se muestran cinco operaciones orientativas de la recepción consideradas como otros tantos momentos de una estrategia comunicativa susceptible de estar al origen de las recepciones observadas.

\section{Abstract}

This article presents our analysis of the permanent exhibition at the Raw Art Museum (musée de l'Art Brut) in Lausanne, Switzerland. This exhibition can be viewed as an communicational artefact that takes on multiple meanings according to the various ways it is received by visitors. Nevertheless, in order for it to be constituted and understood, this artefact should include a series of operating instructions. The author, an informed visitor, tried to bring out its structure by interpreting data on both production and réception of the exhibition. She underscores five directional operations of reception; these may be regarded as a so many phases of a communications strategy that could be one of the foundations of vistors' interpretations.

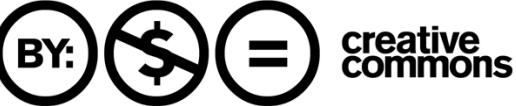




\section{LA STRATÉGGE \\ COMMUNICATIONNELLE \\ DU MUSÉE DE L'ART BRUT: \\ CONSIDÉRATIONS \\ D'UN RÉCEPTEUR AVISÉ}

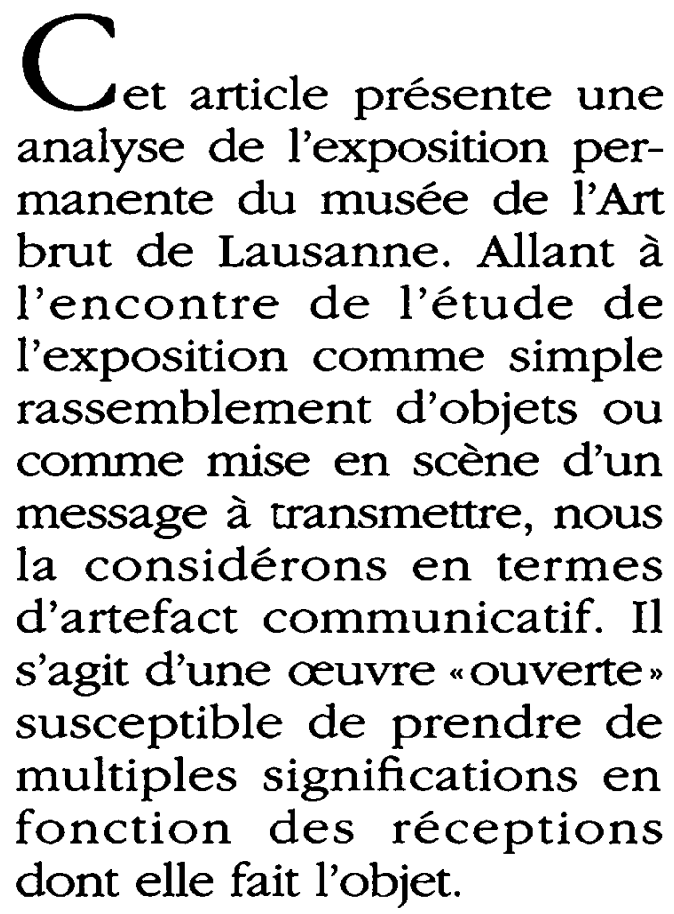

Mais, à son tour, l'exposition se sert de dispositifs orienteurs qui guident les visiteurs dans leur travail d'attribution de sens. Nous tenterons de dégager la structure de cette œuvre par l'interprétation de données faisant référence autant à sa production qu'à sa réception.

L'exposition qui nous occupe accueille la collection d'objets d'art brut constituée à partir des années quarante par l'artiste français Jean Dubuffet. Il s'agit d'objets créés principalement par des personnes dépourvues de formation artistique et vivant dans des situations de marginalité: malades mentaux, prisonniers, vagabonds [...] Le présupposé qui organise la sélection et la réunion de ces productions est qu'un objet devient une véritable ouvre d'art du moment où "... il livre d'une manière très véridique et immédiate — tout chauds, pourrait-on dire, et tout crus - les mouvements d'humeur de l'auteur..." (Dubuffet, 1967, p. 206). Cette expression intérieure pouvant surgir plus facilement quand l'individu est libéré de toute contrainte culturelle.

À l'image de l'immédiateté de la création de "l'œuvre brute", le conservateur du musée ${ }^{1}$ qualifie de contact direct la rencontre du visiteur avec les objets présentés. Il fait ainsi l'impasse sur l'exposition comme médiation. Notre travail, dont cet article présente une partie ${ }^{2}$, prétendait approfondir la connaissance de l'exposition par la mise en évidence des opérations nécessaires, tant de la part des producteurs que des récepteurs, à l'établissement de la compréhension entre l'œuvre et le visiteur. 
Les réceptions du musée, que nous avons présentées dans un autre texte (Gonzalez Martinez, 1995), se caractérisent par leur complexité et leur diversité. Néanmoins elles présentent, pour la plupart, un trait commun qui est le sentiment de consonance entre le musée et le visiteur. Que les réceptions se développent sous les signes de la jouissance esthétique, du bouleversement affectif ou de la critique d'un art de la souffrance, elles impliquent toutes un sentiment de compréhension. Avoir compris signifie, pour le visiteur, réussir à lire les ouvres. Pour le faire, le public emprunte largement la clé d'interprétation que le musée lui offre: les objets montrés sont des reproductions de l'intériorité et du vécu de l'artiste.

L'objet de notre article n'est pas d'expliquer les réceptions observées en les rattachant à des éléments muséologiques. Nous prétendons, plutôt analyser le musée en tant qu'outil de communication afin de faire par la suite une hypothèse sur la relation entre la structure de l'artefact-exposition et la satisfaction observée chez les visiteurs.

Plus en avant dans notre texte nous traiterons de la différence entre les contenus que le concepteur cherche à transmettre et le sens virtuel de l'artefact-exposition. L'objet soumis à la réception n'est pas l'exposition telle que le conservateur a eu l'intention de la réaliser, mais telle qu'elle se présente aux yeux des visiteurs. Il en découle la difficulté de réaliser une analyse objective de l'exposition, indépendante de toute réception. Il est ainsi parce que toute description est déjà une réception qui occasionne une sélection et modifie la réalité de l'exposition. Il s'impose donc d'éclaircir la nature de l'analyse du musée que nous proposons par la suite, de façon à la distinguer d'une simple présentation d'impressions subjectives.

Pour effectuer cette analyse nous nous basons sur des données faisant référence autant à la production qu'à la réception du musée.

En ce qui concerne l'exposition, notre matériel empirique est formé de deux entretiens avec le conservateur du musée et de multiples échanges informels avec ses collaborateurs. Ces conversations portaient sur les idées fondatrices de la collection, le processus de création du musée, ainsi que sur le projet muséologique. À ces témoignages verbaux s'ajoute un travail documentaire sur la constitution, et de la collection et du musée 3 . En outre, nous avons complété la description de l'exposition donnée dans différents documents par une analyse des textes adressés aux visiteurs ainsi que par des observations réalisées sur place. Notre objectif était d'identifier l'interprétation des œuvres proposée par le musée et de saisir la mise en scène développée afin de présenter les objets artistiques et les contenus théoriques.

Nous avons étudié la réception de l'exposition à l'aide de vingt entretiens semi-directifs, avec autant de visiteurs, et de l'analyse du livre d'or du muséét. Dans ce texte nous ne présenterons que sommairement ces données. Néanmoins, notre analyse de l'exposition en tant qu'ensemble de dispositifs d'orientation de la visite s'ancre sur ce travail préalable d'étude de la réception. Ce sont seulement les propos des visiteurs qui permettent d'élucider comment l'exposition agit de fait sur les individus. 
Une fois abandonnée toute ambition d'exercer une médiation transparente entre la réalité et son récit, il semble légitime de joindre aux contributions de notre travail notre propre réception. L'œil qui observe est un oil informé par toutes les données recueillies ainsi que par un certain habitus social et sociologique. C'est dans ce sens que nous parlons d'un visiteur avisé.

Dans un premier temps, nous présenterons le musée de l'Art brut. Nous énoncerons les fondements théoriques liés autant à la création de la collection et du concept d'art brut qu'à la production du musée. Après cela, nous ferons une brève description de la matérialité de l'exposition.

Dans un deuxième temps, nous énoncerons et appliquerons à l'étude de l'exposition quelques concepts provenant de l'analyse littéraire. Ceci nous permettra de développer une réflexion sur la nature de l'objet-exposition et sur le travail de production et de réception de celuici.

Par la suite, nous nous pencherons sur les moyens utilisés par les concepteurs de l'exposition pour mettre en scène les ouvres. Pour le faire, nous dessinerons le modèle muséologique dans lequel s'insère le musée de l'Art brut. Il s'en suivra la présentation de la stratégie communicationnelle du musée.

En conclusion, nous nous demanderons comment la structure de l'exposition est susceptible de donner accès à un certain type de réception.

\section{DE L'OBJET BRUT AU \\ MUSÉE DE L'ART BRUT}

THÈMES LIÉS À LA CRÉATION DE L'EXPOSITION

Dans les années quarante Jean Dubuffet entreprend, notamment dans les hôpitaux psychiatriques, la recherche d'objets bruts. Son labeur de collectionneur s'accompagne de celui de théoricien de l'Art brut de façon à forger ce concept et doter sa collection d'une identité propre. En 1972, Dubuffet fait donation à la ville de Lausanne de la collection qu'il a créée. Les objets avaient été conservés à Paris à l'exception d'un séjour à New York. Depuis son arrivée à Lausanne, la collection s'est enrichie considérablement, au point que les œuvres cédées par Dubuffet représentent actuellement moins d'un tiers du nombre total d'objets conservés dans le musée. Néanmoins, l'expansion de la collection se poursuit toujours selon les critères posés par son créateur.

La création de la collection est liée à la situation artistique de l'époque. La relation entre l'art et l'aliénation mentale et la culture comme contrainte à la création, sont deux idées au coeur des mouvements intellectuels du début du siècle. Les idées anticulturelles furent soutenues par des mouvements tels que le Dadaïsme et le Futurisme, ainsi que par les cercles surréalistes que Jean Dubuffet fréquentait à Paris dans les années 1920. André Breton lui-même était membre fondateur de la Compagnie 
de l'Art brut. La création de la collection d'art brut se situe parmi les efforts qui se développèrent au xxe siècle pour retrouver une créativité pure et libre.

La collection est, également, en étroite imbrication avec la trajectoire personnelle et artistique de Dubuffet. Autant par ses réalisations pratiques que par ses écrits, l'artiste mène une lutte en faveur de l'art dégagé de toute contrainte esthétique et culturelle. En fait, Dubuffet arrive à imposer sur le marché sa production et celle des artistes bruts par une démarche commune de retour aux sources. "C'est seulement quand j'ai entrepris de pousser plus loin la renonciation à tout ordre esthétique et quand il m'est advenu d'éprouver sur moi-même les effets qu'il était possible d'obtenir à la faveur d'ouvrages procédant de cette totale renonciation que j'ai pris conscience de l'inanité des préoccupations esthétiques et des possibilités de la voie dans laquelle je me trouvais dès lors engagés."

Le parcours personnel et professionnel des différentes personnes impliquées dans la création de l'art brut pourrait nous fournir une bonne grille d'analyse des thèmes de l'exposition (Thevoz, 1986; Solomon, 1973). Ici, nous nous limiterons à présenter les idées principales développées par Dubuffet au sujet de l'art brut qui sont à la base de la création de la collection et de sa mise en exposition à Lausanne.

La collection d'œuvres exposées au musée de l'Art brut s'organise sur quelques lignes directrices. Il se trouve parmi celles-ci la relation entre l'art et l'aliénation mentale et la conception de la culture comme contrainte à la création.

Dubuffet était convaincu que chacun pouvait développer une activité créative et que cette fonction humaine, considérée comme une opportunité de liberté, a été anéantie par la civilisation, par l'éducation et par l'importance donnée à la technique et à la juste représentation de l'objet (Dubuffet, 1968).

Sa conception de l'art l'amène à réfuter l'idée de l'art et de la beauté telle qu'elle est partagée par la société occidentale, c'est-à-dire l'art dépourvu de ses fonctions magiques et spirituelles, devenant objet de décoration et d'arrangement de formes et de couleurs "... pour un prétendu plaisir des yeux" (Dubuffet, 1967, p. 99). L'Art brut se caractériserait, au contraire, par son originalité, par son caractère "spontané et inventif, non mimétique "(Dubuffet, 1968).

Des écrits de Dubuffet découle un effacement des limites, d'une part entre la folie et la création et d'autre part entre la normalité et la folie. Ainsi, le travail de création serait un acte, impliquant une extrême tension, qui ne peut jamais être considérée comme normale. Et la folie peut être vue comme l'expression d'une révolte face aux contraintes imposées par la civilisation (Dubuffet, 1967, p. 455).

En conclusion et par opposition aux productions des savants et des vedettes de l'art, Dubuffet va donner de l'importance aux productions des individus qui pour une raison ou une autre "... ont échappé au conditionnement culturel..." (Citation tirée du panneau introductif situé à l'entrée de l'exposition). Ce serait parmi les productions de ces hommes, l'bomme $d u$ commun, que se trouverait la vraie création. La recherche d'images 
non conditionnées par la culture occidentale conduit Dubuffet à s'adresser à des personnes étrangères aux milieux artistiques, victimes de l'exclusion ou des personnes n'ayant pas reçu une formation scolaire. Hors de la vie en société, les objets manifesteraient le vécu de l'auteur immergé dans l'exploration de son intérieur.

En ce qui concerne la conception de l'exposition, les propos du conservateur ${ }^{6}$ se basent sur une opposition par rapport aux musées d'art culturel. L'art officiel s'évertue à "agenouiller les gens" (Thevoz, 1985, p. 7). C'est une relation de savoir et de pouvoir qui débouche, dans le meilleur des cas, sur un simple plaisir esthétique. Par contre l'exposition qui nous occupe cherche à établir un rapport actif entre les ouvres et le visiteur et non une relation unilatérale. Le but poursuivi est de développer la capacité inventive du spectateur et de parvenir à ce qu'il s'engage dans la construction d'un sens.

Le concepteur de l'exposition envisage la visite comme l'établissement d'un "contact intime le plus immédiat possible" entre l'œuvre et le récepteur. La relation avec l'art brut passe par l'émotion. Il s'agit de favoriser un contact émotionnel fait "de peur, d'inquiétude et d'angoisse".

L'exposition, malgré l'absence d'encadrement didactique, vise à "emmener le visiteur à découvrir et à développer une problématique" (Thevoz, 1989, p. 36). Le concepteur cherche, au-delà de la transmission des informations ou de la jouissance esthétique, à provoquer une prise de conscience. Du rôle de "révélateur des idées informulées" découlerait la capacité de l'exposition de déclencher un processus de réflexion afin que le visiteur ressorte modifié de l'exposition (Thevoz, 1985).

Le motif autour duquel s'articule le discours du concepteur en référence au travail de création de l'exposition, est la non-ingérence. Il fait souvent référence aux œuvres comme étant la seule cause des formes que prennent autant l'exposition que les réceptions de celle-ci. Le concepteur fait l'éloge des textes sobres et objectifs qui accompagnent les œuvres. Quant à la réception, il s'agirait de ne pas attribuer au décor, dont on revendique l'aspect neutre, des réactions provoquées par les caractéristiques formelles des objets.

\section{L'EXPOSITION DANS TOUTE SA MATÉRIALITÉ}

Le musée de l'Art brut prend place dans quatre corps de bâtiments distincts, quoique unis, du château de Beaulieu: la grange, où est montré l'essentiel de la collection, l'aile sud-ouest, l'annexe-nord, aménagée pour accueillir les visiteurs et le corps central du bâtiment.

Ce qui fut la grange est un vaste volume clos, divisé en quatre niveaux. L'intérieur est organisé autour d'un puits central «... de manière à laisser passer le regard et à donner ainsi au visiteur la vision du volume entier ${ }^{7}$. Un jeu d'escaliers lie "... un cheminement logique permettant une visite systématique des espaces ". Ces derniers sont créés et transformés grâce à un système de panneaux et de vitrines mobiles, telles des alvéoles, où sont exposées les œuvres d'une taille mineure. Dans la 
construction intérieure, il a été fait usage de matériaux et de couleurs absorbant la lumière et les sons; plafonds, sols et murs sont noirs.

Des espaces réservés aux expositions temporaires sont situés dans l'aile sud-ouest, au rez-de-chaussée du corps central et dans la partie sud du premier étage. Dans cette dernière, le visiteur peut visionner des cassettes vidéo concernant l'art brut. Dans ces deux salles aux murs blancs, des panneaux recouvrant les fenêtres tamisent la lumière extérieure.

Dans la partie destinée à l'accueil des visiteurs, à côté de la porte d'entrée de la salle d'exposition, se trouve un panneau divisé en cinq colonnes. Le contenu de ces colonnes est constitué de : cinq photos montrant des vues du Foyer de l'Art Brut à Paris en 1970; un historique de la collection de 1945 à 1975; une photo de Dubuffet et en dessous une reproduction d'un de ses textes manuscrits, sur sa conception de l'art; un texte titré "Qu'est-ce qu'est l'art brut?" avec comme sous-titres: "Ne pas confondre!", "L'art naîf ", "L'art des fous", "Les arts primitifs"; un texte titré "Pressentiments" composé de déclarations de Champfleury, Léon Tolstoi, Ferdinand Cheval, Auguste Marie (psychiatre), Paul Klee, faisant référence directe ou indirecte aux ceuvres de l'Art brut.

À l'intérieur, l'accrochage est très serré. Les objets, tableaux, sculptures, dessins, se présentent selon une grande variété de formes, grandeurs et matériaux souvent novateurs par rapport à ceux des objets qu'on peut observer dans les musées d'art conventionnels. Les œuvres, regroupées, sauf quelques exceptions, par auteurs, sont accompagnées d'une notice biographique et d'une photo de l'artiste. Les tableaux ne sont pas accompagnés des titres. Il n'y a pas de fiche muséologique concernant la réalité matérielle de l'objet, support, dimensions ou date de création.

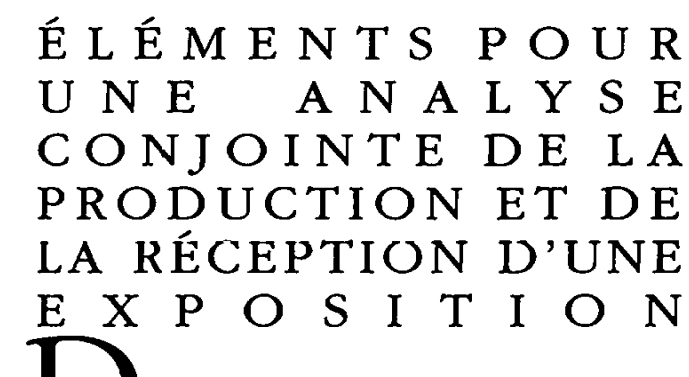

ans les conceptions courantes de la communication littéraire, le sens d'une ouvre est déjà présent et la lecture ne fait que le découvrir plus ou moins entièrement. "Lire n'est pas produire, mais reproduire" (Rutten, 1980, p. 72). Cette conception de l'œuvre implique une théorie du texte reposant sur "... une négation du sujet en tant qu'instance de production de sens..." (id., p. 73). Ainsi tout ce qu'un lecteur pourra relever du texte au cours d'une lecture n'a rien de nouveau. Ce n'est, dans le meilleur des cas, qu'une partie de son essence.

Tout autrement, l'École de Constance postule que l'œuvre est une structure de signes de nature purement matérielle, un artefact dépourvu d'un sens en soi ou d'un sens préexistant à sa lecture. Cette structure, pour être constituée et comprise, formule des directives à suivre. Le texte autorise différentes marges de manœuvre à ses lecteurs et préoriente ses 
lectures à l'aide des signaux, des instructions ou des consignes de décodage. De tels éléments, constitutifs de l'œuvre, ont la fonction spécifique d'informer et, par ailleurs, de stimuler le lecteur à donner un sens à ce qu'il perçoit.

Les textes littéraires sont des textes de fiction. Les objets représentés dans l'œuvre se caractérisent par le rapport qu'ils entretiennent avec la réalité désignée. Ceux-ci sont esquissés sans arriver à reproduire une réalité existante. Le texte, pour ces raisons, sera qualifié de configuration schématique comprenant des lieux divers d'indétermination. Les lieux d'indétermination se présentent au lecteur comme des vides, blancs, que celui-ci est appelé à combler. Ces vides déclenchent son imagination et vont être remplis à l'aide des représentations. Au cours de la lecture se développent dans la conscience du lecteur des images qui témoignent de l'effort fait pour se représenter ce qu'il ne peut pas voir (Iser, 1985). Il se forme finalement dans l'esprit du lecteur une configuration spécifique de contenus, appelée "concrétisation de sens". Cette concrétisation est le résultat de l'agencement des significations produites par l'individu au cours de sa lecture.

L'œuvre serait la résultante de la convergence de l'artefact et de sa réception. Donc, de l'action du texte, de l'effet qu'il produit et de la réception que lui réserve son destinataire.

Nous venons de présenter quelques éléments théoriques sur lesquels se base notre travail. Maintenant nous nous interrogerons sur la nature de l'exposition en tant qu'artefact, sur sa production et sur sa réception.

Couramment, on entend par exposition un espace aménagé où un ensemble d'objets organisés autour d'un thème sont montrés à un public9. La production d'une exposition présuppose l'existence d'un regard constitué à partir d'une idée qui opère la sélection des objets et qui, en les lisant, leur donne un sens qui justifie l'ensemble. Ce travail de création, mené à terme par l'auteur de l'exposition, dont résultera un objet différent de l'ensemble d'éléments qui le composent, nous autorise à parler de l'exposition en tant qu'œuvre. Une telle analogie nous permet de transférer des concepts développés dans le domaine littéraire à l'étude de la réception d'une exposition ${ }^{10}$.

La conception et la réalisation d'une exposition supposent différents moments. D'abord une sélection et une accumulation d'objets extraits du monde réel. En l'occurrence, en ce qui concerne notre musée, des "... ouvrages exécutés par des personnes indemnes de culture artistique" (Dubuffet, 1967, p. 201). Dans le cas de l'Art Brut, nous pouvons parler de création parce que Dubuffet ne se limite pas à établir entre les objets réunis des rapports déjà existants et reconnus, mais des rapports nouveaux. La démarche qui préside à la collection définit "... un nouveau territoire, celui de l'art brut. Territoire qui apparaît soudain sur les cartes; avant c'était zone blanche, terra incognita. Il n'y avait que des œuvres isolées, objets de curiosité. Groupées, ces œuvres prennent un autre sens, s'éclairent l'une l'autre" (Soisson, 1973, p. 179). 
Toutefois cette accumulation demande une justification. Elle nécessite l'existence d'un regard constitué à partir d'une idée. Ainsi, le musée est une construction idéologique, qui vient donner une raison à l'ensemble d'œuvres et les convertir en objets de musée. À l'origine de la collection de l'Art Brut, nous trouvons un système d'idées qui s'élabore à partir d'une confrontation des objets. Depuis 1947, Dubuffet entreprend un travail de théoricien de l'art brut. Il s'agit de fournir à la collection une esthétique et d'établir les fondements logiques à l'existence de celle-ci arrivant ainsi à devenir l'inventeur d'un art. Ce système d'idées, une fois bien constitué, est mis en place au moment de la création de l'exposition et projeté sur les œuvres figurant comme illustrations de ces idées ${ }^{11}$. La création d'une collection suppose, ainsi, une lecture des objets à travers laquelle un sens est donné à l'ensemble.

Si nous reprenons notre analogie avec le texte, nous pouvons dire que la collection est une concrétisation qui, ensuite comme résultante d'un nouveau processus de lecture, va devenir musée. Le sens donné à la collection est extériorisé en forme d'exposition. Son concepteur réunit et sélectionne, à nouveau, les objets. Il les accompagne de textes et de photos et à l'aide d'une série d'outils d'exposition, panneaux, vitrines, dispositifs d'éclairage, procède à leur mise en scène.

La conception d'un musée inclut une mise en parcours de l'exposition et, tel un "livre épinglé" (Clement, 1983, p. 39), elle se construit autour d'un thème développé et traduit en termes d'accrochage et de distribution des objets dans l'espace. Il apparaît donc clairement que l'exposition est une œuvre et que son élaboration débouche sur un produit différent de l'ensemble des objets. De cette façon, nous ne considérons pas l'exposition comme traduction transparente qui se limite à montrer l'essence interne des ouvres, ni comme une construction qui trahirait la véritable nature de celles-ci. Il s'agit d'une œuvre à objectifs définis qui vise à présenter un thème, une idée. L'artefact-exposition créé par les concepteurs a pour objet, entre autres, d'orienter la lecture des artefactsobjets qui sont montrés conjointement à ses instructions de lecture. Le discours qui a déterminé le choix des objets et la mise en exposition s'inscrit dans l'espace, dans les objets nouvellement créés et susceptibles d'acquérir une signification différente. Sa trace peut être perçue dans les éléments mis en ouvre au sein du monde nouveau que constitue l'exposition, le "monde synthétique".

Dès que la définition de l'objet-exposition, différencié de l'ensemble des œuvres, s'impose et du moment où l'intérêt du musée consiste à proposer une interprétation des œuvres qu'il présente, alors devient évidente l'importance de l'activité de conception de l'exposition. L'exposition ne se produit pas suivant les lignes directrices d'un texte, mais il s'agit plutôt d'une série de négociations, d'interactions et de contraintes qui façonnent l'objet final. Le concepteur et le réalisateur de l'exposition ont pour labeur la construction et la projection en forme d'exposition d'un discours dirigé vers le public.

Nous voyons donc comment l'exposition est une concrétisation qui s'inscrit à l'intérieur d'une chaîne de concrétisations. L'élaboration d'une 
exposition, telle que l'Art brut, résulte de la coopération de plusieurs auteurs. Si nous considérons l'exposition comme une série de tableaux, le premier auteur est le producteur matériel de ceux-ci. Le collectionneur qui a sélectionné et accumulé les objets et le théoricien qui établit des rapports nouveaux entre les objets, sont des auteurs nouveaux. La sélection des objets et leur mise en scène ont pour artisans le concepteur et le réalisateur de l'exposition. À chaque sélection ou lecture, l'objet ressort modifié. Quand le visiteur reçoit l'exposition il se situe, lui aussi, dans cette chaîne de concrétisations.

Exposer ne signifie pas montrer l'objet et donner accès à son sens inhérent. Mais “... exposer c'est, toujours et inévitablement, proposer, de ce que l'on montre, un sens particulier". De cela découle la nature de l'activité du visiteur, "...visiter une exposition c'est com-poser" (Veron \& Levasseur, 1983, p. 21). Accommoder, s'approprier, négocier un rapport avec l'énonciateur de l'exposition et avec ce qu'il expose.

Dès le moment de son entrée au musée, le visiteur réalise une série d'opérations de lecture qui lui permettent de lire l'exposition. Le spectateur sélectionne des éléments des objets et de l'exposition. Ces éléments deviennent par la suite motifs d'appel et d'attribution d'un sens. Le visiteur réalise un travail cognitif d'élaboration et de combinaison des données. Il s'interroge, se répond et lance des hypothèses. Ce travail lui permet de donner une cohérence à ce qu'il voit et de poursuivre sa lecture.

Par ailleurs, la lecture réalisée par le visiteur n'est pas un simple enjeu de facteurs subjectifs. Chaque récepteur détermine le sens de l'œuvre, orienté par les normes et mesures de valeur spécifiques de sa société et de l'histoire artistique de son groupe. Avec un regard qui oscille entre le moi et l'objet, le visiteur fonde, plus ou moins inconsciemment, sa lecture sur l'ensemble de son expérience.

Le visiteur, "... de par la vision successive des œuvres, de par leur rassemblement et de par l'environnement qui les accompagne..." (Davallon, 1986, p. 259), produit une concrétisation de sens, ce que Davallon appelle "le monde utopique". L'ensemble des opérations de lecture peut être compris comme un travail d'appropriation destiné à réduire la "différence" entre l'œuvre et son lecteur. La lecture d'un public non spécialiste ne constitue pas un acte où le lecteur tente consciemment de découvrir le code utilisé dans la production de l'œuvre afin de la déchiffrer (Bourdieu, 1992, p. 431-441). Il ne s'agit pas non plus, seulement ou forcément, d'une absorption passive, mais plutôt d'un travail voué à rendre semblable l'objet "... à ce qu'on est, le faire sien, se l'approprier" (de Certeau, 1990, p. 241). La lecture vise à rendre les objets assertoriques (Passeron, 1991), non seulement dans le sens de leur faire dire quelque chose, mais pour pouvoir s'ancrer en eux et établir une interaction.

Nos affirmations vont à l'encontre de l'application simpliste de la théorie de l'information à l'étude de la réception. Celle-ci supposerait, si nous parlons d'une exposition, que la visite constitue un moment où s'établit une communication à sens unique entre un émetteur et un récepteur visant à transmettre un message défini. Adopter ce point de vue 
mène à étudier la réception sous l'angle du décalage et des absences relatives aux contenus émis par l'auteur de l'exposition, ou par rapport au sens univoque que l'œuvre proposerait. Nous postulons, au contraire, qu'il est impossible de déduire les effets de ce qui est exposé. Notre perspective s'oppose à l'idée que l'intention de l'œuvre coïncide avec l'intention de l'auteur et que l'exposition ne peut dire que ce que son concepteur a voulu qu'elle exprime.

L'énonciation de la production et celle de la réception, s'articulent sur un double rapport (Davallon, 1986, p. 252) : le rapport d'emboîtement et le rapport de développement. En contemplant les objets, le visiteur les lit au travers et dans l'exposition. D'une certaine façon, les objets sont susceptibles de prendre une signification différente par le fait d'être placés à l'intérieur de l'exposition. Celle-ci le conduit dans sa façon d'aborder les objets et l'incite à faire appel à un certain type de connaissances. C'est dans ce sens que l'on peut dire que l'exposition médiatise la relation entre le visiteur et l'objet.

Toutefois, nous parlons de rapport de développement parce que ce cadre n'est pas contraignant au point de rendre l'individu passif. L'exposition ne détermine jamais un sens mais définit un éventail d'effets de sens possibles. Elle est à considérer comme une complexité de sens potentiels qui vont être mis en mouvement par le visiteur. Une sorte d'offre avec laquelle le visiteur négocie afin de construire son sens.

Par "lecteurs", Jauss entend trois groupes distincts: les lecteurs normaux qui simplement lisent et réalisent une réception passive, les lecteurs-auteurs incités par l'œuvre à produire à leur tour, et les lecteurs actifs (les critiques et les interprètes) pour qui l'œuvre est objet d'examen ${ }^{12}$. L'auteur de cet article prétend se rallier du côté de ces derniers. Il s'agit d'un récepteur averti qui se sert de sa réception et de celle des visiteurs, tout comme de son interprétation des diverses données recueillies, pour étudier la structure du musée. Son écrit vient, ainsi, se situer dans la chaîne de concrétisations de l'objet exposition.

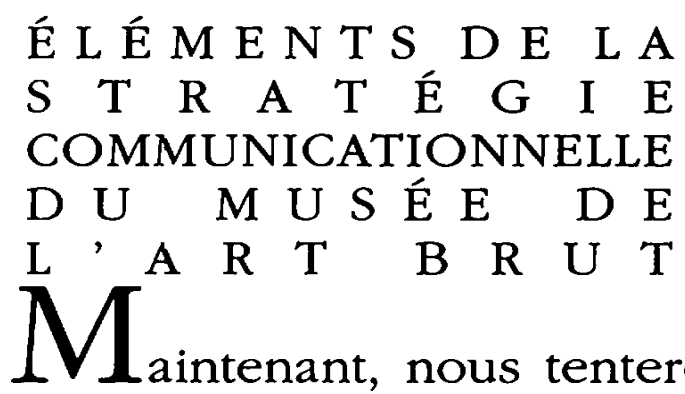

ture de l'objet-exposition et de mettre en évidence la logique qui préside à la conception et la réalisation du musée. Il sera question des moyens utilisés par les concepteurs pour accompagner les objets, ainsi que des éléments de l'exposition qui médiatisent la relation du visiteur aux œuvres. Nous resterons attentif au développement de cette stratégie communicationnelle, dans la mise en forme des objets et des contenus présentés. Une analyse approfondie de l'exposition se déploierait à la frontière entre la sociologie de l'art et la sémiologie. La difficulté de ce 
genre d'études est considérable si on tient compte du nombre d'éléments qui forment l'exposition, de leur nature composite et du fait qu'ils appartiennent à des systèmes symboliques très variés.

La nature de notre analyse découle des présupposés théoriques que nous avons annoncés antérieurement. L'exposition est pour les concepteurs un outil communicationnel, mais elle transcende sa nature de média pour abriter une complexité de sens possibles. Nous rappellerons aussi que l'exposition ne se produit pas suivant les lignes directrices d'un texte, mais qu'il s'agit plutôt d'une série de négociations, d'interactions et de contraintes qui façonnent l'objet final13.

D'un point de vue général, le musée de l'Art brut s'insère dans le nouveau modèle muséologique qui cherche à faire éprouver des émotions au visiteur en vue de le séduire (Perraton, 1987). En suivant la classification des expositions de Jean Davallon, l'exposition de l'Art brut peut être étudiée sous un double aspect (Davallon, 1986, "Avant-propos"). D'une part, il s'agit d'une exposition d'art du même type que celles qui ont lieu dans les musées traditionnels. L'objectif premier de ces expositions est de permettre au visiteur de voir les objets, de favoriser une situation de rencontre entre visiteur et ouvre. D'autre part, cette exposition est un outil pour établir une communication. Les objets sont situés à l'intérieur d'une exposition qui joue un rôle déterminant dans l'interaction entre objets et visiteur. Il s'agit d'une exposition qui, au travers des dispositifs de mise en scène, cherche à montrer, décrire et mettre en valeur, idées et objets, afin d'assurer l'établissement de la communication.

Il est difficile de comprendre la production du musée de l'Art brut sans prendre en compte les changements survenus dans le monde de l'exposition depuis les années cinquante. La Nouvelle Muséologie développée après la guerre met l'accent sur les fonctions de communication des musées afin de les ouvrir à un public plus large et de leur donner un rôle actif dans la société. Le renouvellement se fait principalement sur deux fronts, au niveau de l'architecture et de la présentation des contenus.

Les changements au niveau architectural se basent sur la récusation des signes du spectacle institutionnel. C'est dans cet esprit que l'on critique l'architecture du "musée-temple": les grands escaliers, les colonnes, les dorures et les planchers en bois. Les architectes et les conservateurs chez qui "... la dénégation s'est imposée comme mode d'énonciation privilégiée..." (Besson, 1986, p. 40) se tournent vers un nouveau modèle muséologique. Celui des espaces neutres, des volumes plus petits, des mobiliers et encadrements fonctionnels.

Quant à la présentation des contenus, le musée-mémoire qui voulait transmettre des connaissances dans un esprit didactique est supplanté par le musée-spectacle. Aux ambitions de la nouvelle muséologie correspond un type d'installation muséale qui cherche plutôt à séduire qu'à convaincre. Les musées traditionnels mettaient en œuvre un appareil didactique voué à faire approuver ce qui était énoncé. Les musées-spectacles cherchent par contre à faire éprouver pour persuader. Afin d'y parvenir, de nouveaux environnements fondés sur la séduction prennent place. Les conservateurs et concepteurs d'expositions font appel à un cer- 
tain nombre de procédés de mise en scène visant à "frapper les sens pour mieux convaincre la raison" (Dagognet cité dans Perraton, 1987, p. 26. L'évolution des musées du "musée-mémoire" au "musée-spectacle" est développée par Perraton, ibid.). Devant les nouveaux musées, telle que la Collection de l'Art brut, le spectateur est devant une hybridation "... entre les techniques muséales et les techniques du spectacle..." (Veron, 1992, p. 34) Toutes ces techniques doivent être considérées comme des outils d'une stratégie de communication.

OPÉRATIONS ORIENTATIVES DE LA RÉCEPTION

Nous avons cerné cinq opérations qui nous semblent composer la stratégie communicationnelle mise en œuvre dans le musée: présentation et durcissement de l'énoncé, cohérence, mystère, réponse et affectivité. Avant de passer à l'étude de ces composants, voici quelques considérations d'ordre général.

L'exposition révèle un équilibre entre la visualisation et l'explication des idées, c'est-à-dire "transmettre des idées avec des choses" et "expliquer des objets par des idées" (Heinich \& Pollak, 1989, p. 31). Le parti de la lisibilité et de l'explication est mené à terme grâce au panneau introduisant le musée situé à l'extérieur de la salle d'exposition. L'intérieur de l'exposition, en privilégiant les objets esthétiques et en introduisant des notes biographiques qui reconstituent le contexte de l'œuvre, prend parti par la visualisation.

En tous cas, il est nécessaire de considérer autant les idées que les objets comme deux systèmes étroitement liés. Les idées viennent s'incarner dans l'espace de l'exposition, les objets placés, les outils d'exposition qui les accompagnent et le parcours à suivre "... et à partir duquel le visiteur sera amené à éprouver physiquement" (Heinich \& Pollak, 1989, p. 64) le thème de l'exposition. Le tout étant disposé de telle façon qu'il illustre un système d'idées.

1) Présentation et durcissement de l'énoncé: L'exposition débute avec un texte introductif. Ce texte vise d'une part à présenter l'énoncé qui va être par la suite démontré et d'autre part à le renforcer. Ce texte, donc, ne se limite pas à transmettre l'information, il agit.

La première procédure empruntée pour persuader le visiteur est la sortie hors $d u$ silence ${ }^{14}$ et la reconnaissance du caractère exceptionnel de l'œuvre. L'auteur du texte procède à une particularisation de l'œuvre en mettant en vue ses caractéristiques stylistiques exceptionnelles et les circonstances de la vie de son auteur. Cette procédure comprend également une généralisation. Le texte tente de construire une cohérence entre l'ensemble des objets afin d'en parler comme d'uun art". Les objets sont rapportés comme un ensemble face "au reste" des productions artistiques.

Par ces opérations le texte crée un ensemble d'objets qui attend une interprétation. Il opère l'ouverture d'un espace berméneutique ("Qu'estce que c'est l'Art brut?". Texte introductif) ${ }^{15}$. Cette interprétation est don- 
née par la suite ainsi que les critères d'appartenance au nouvel art ("Ne pas confondre! L'art naïf. L'art des fous. Les arts primitifs". T.i.). Ce texte introductif sous la forme d'une mise en abîme offre à l'intérieur de l'exposition sa reproduction. Il facilite la compréhension de l'ensemble et procure au visiteur le point de départ de sa lecture. La première pièce de cet emboîtement d'éléments est le nom du musée. L'exposition va être successivement reprise dans son entier par le titre, la déclaration de Dubuffet et le panneau introductif. Ce texte indique, donc, le mode de lecture à adopter ("il faut pénétrer profondément dans ces embryons rudimentaires et laisser de côté...". T. i.) et guide la réception en préparant le visiteur à ce qu'il va voir ("Ici, nous entrevoyons des humeurs et des sentiments...". T. i.).

De même que le concepteur rencontre des difficultés à procurer au visiteur l'entier des informations dont il dispose, il lui est impossible de créer un écrit ne révélant pas une idéologie. Les textes dont nous parlons sont des textes "nettoyés" et prêts à entrer dans l'argumentation. Ceci est présent dans la procédure de "durcissement" exposée ci-dessous : $L a$ mise en bistoire.

En effet, l'historique part du texte introductif, présente la création de la collection comme une découverte d'objets par nature bruts ("premières recherches d'art extra-culturelles". T. i.). En réécrivant l'histoire de l'Art brut et en passant sous silence sa préhistoire, le texte signale qui sont ses fondateurs et ses autorités indiscutables. Également, l'historique sert à décrire les méthodes de la découverte de l'Art brut; méthodes dont la pureté est garante de l'authenticité des objets.

L'enjeu du texte introductif est l'autorité. Il ne suffit pas de donner une définition mais il est nécessaire d'accumuler assez d'autorité pour la rendre crédible. Ce texte argumente, en excluant les opinions divergentes et en faisant appel aux alliés. Les citations de personnages célèbres qu'inclut le texte introductif augmentent le crédit du musée et attestent de la valeur des œuvres montrées. La même fonction est remplie par le témoignage de Dubuffet. Sa déclaration manuscrite, sa présence par sa photographie et sa signature attestent l'authenticité de l'exposition.

2) Cobérence: L'idée qui régit la mise en exposition est de former une réunion d'objets hétérogènes en un tout cohérent afin de pouvoir en parler en tant qu'un art. Les deux pôles de ce travail sont la quête de l'unicité et de la globalité. Le musée établit des rapports de différence et de similitude entre les objets. Par la sélection et la disposition d'objets que la création du musée comporte, les œuvres deviennent uniques par rapport à celles de l'extérieur et différentes entre elles afin de rendre visible leur caractère personnel. Finalement il fait de cette réunion d'objets un ensemble cohérent.

L'analyse de l'exposition ne révèle pas exclusivement la cohérence entre objets mais également l'identification des objets exposés avec le cadre-musée. Nous pouvons dire qu'en général tend à être gardé un parallélisme entre les traits distinctifs des objets et les caractéristiques formelles de l'exposition. Pour ne citer qu'un exemple, le caractère bigarré de la plupart des objets se reproduit dans un accrochage très serré. 
Il est possible d'apprécier, également, une forte identité entre les caractéristiques stylistiques des objets, la notice biographique et la photo de l'auteur. Cette similitude entre différents éléments contribue à rendre possible "l'interprétance interne "16 de l'exposition.

La cohérence entre des éléments qui s'appellent et s'expliquent réciproquement constitue une matérialisation du lien qui unit les divers objets exposés et simultanément un dispositif de guidage de la réception.

3) Mystère: La stratégie communicationnelle est composée d'une série de procédures adoptées par l'exposition afin d'orienter sa réception. Si la stratégie mise en place impose "... trop clairement au lecteur sa réaction, celle-ci sera obligée, et le lecteur risque d'autant plus de se détourner de ce vers quoi le texte avait voulu l'orienter" (Iser, 1985 p. 163).

Afin de ne pas paralyser l'imagination du visiteur avec des contenus textuels trop déterminants, le musée procède à une mise-en-énigme. Il s'agit de présenter les œuvres comme posant une question dont la solution concerne le public. Le mystère qui inquiète le visiteur et aiguise son désir de comprendre porte sur la vie de l'artiste ("On sait seulement de Bill Traylor que...". Bill Traylor), la production de l'objet ("... cru entendre dans la mine, à l'âge de 35 ans, des voix lui prescrivant de peindre". Augustin Lesage) et son interprétation ("... grand nombre de feuilles porteuses de partitions de musique bellement décorées que nul jusqu'à présent n'est parvenu à lire ". Adolf Wölfli).

Cette énigme n'apparaît pas exclusivement au niveau des éléments textuels mais elle se traduit aussi architecturalement. "Il ne faut pas qu'elle (la collection) se présente comme dans un musée ordinaire, n'estce pas, il faut lui préserver son côté secret, il faut laisser aux visiteurs l'impression qu'ils découvrent quelque chose. Le musée se présente comme une sorte de grande coquille absolument noire avec des petites alcôves et l'accrochage est très serré. Je crois que chacun ira là-dedans comme une aventure." (Thevoz, 1974, p. 102.)

4) Donner la réponse: À travers une mise en légende biographique, l'exposition donne une réponse à la question que posent les objets par leur présence. Le récepteur trouve dans la note biographique l'outil qui permet de parler de l'objet et de le faire parler. Ce n'est pas parce que l'Art brut est plus immédiat que d'autres que le visiteur en parle plus aisément, mais parce que le visiteur possède ou arrive à se procurer les instruments nécessaires à le lire. Un de ces outils est la note biographique; il s'agit d'une légende qui signale ce qu'il faut voir dans l'œuvre: "une voie de sublimation de son agressivité " pour les peintures de Franca Settembrini ou bien "des histoires [...] et des souvenirs de sa vie à la plantation Traylor". D'autre part, les textes servent à montrer quels sont les critères choisis pour inclure les objets dans la collection. Les informations mentionnées deviennent par la suite des principes légitimes d'identification des œuvres. L'absence de titre qui rend l'œuvre plus secrète permet de croire, également, que la réalité représentée n'est pas autre que celle décrite dans la note.

La structure discursive des notes est simple: il s'agit d'un récit chronologique de la vie des auteurs. Ces notes anonymes utilisent souvent la 
forme impersonnelle ("On raconte que...". Scottie Wilson). Tel que dans le langage en sciences humaines, "L'énoncé produit [...] est détaché [...] de l'instance de l'énonciation pour apparaître comme le discours de non personne, n'appartenant à personne, c'est-à-dire comme le discours objectif dont le sujet serait la science se faisant d'elle-même"17. Ainsi les textes sont enveloppés d'une aura de neutralité et transmettent l'idée qu'il n'est pas nécessaire de posséder un code pour comprendre des ouvres qui énoncent d'elles-mêmes leur vérité. Cette construction de l'exposition sur la négation du code permet la défense d'une lecture immédiate et non interprétative des objets et constitue en fait un code en elle-même.

5) Affectivité: À l'absence du concepteur de l'exposition correspond une hyper-présence de l'auteur de l'œuvre. Les textes sont structurés selon un dispositif narratif précis afin de mettre en scène des personnages exceptionnels sous un angle qui les rend familiers ("Ceux qui l'ont connu alors rapportent que, sans détester les hommes, il paraissait préférer la solitude ou plus précisément la compagnie exclusive de son chien, qu'il ne quittait jamais". Carlo). Le musée en procédant ainsi rend possible chez le visiteur un sentiment de sympathie envers les auteurs et l'introduit dans un cercle affectif. D'objet en objet, l'affectivité du visiteur est appelée. Équivalentes au rituel de présentation d'un individu, ces notes empêchent le visiteur de prendre de la distance par rapport à la vie de l'artiste. En contextualisant les objets, l'exposition devient une machine à sentir. $\hat{A}$ travers les notes biographiques et les photographies, principalement si elles reproduisent l'œuvre à côté de son auteur, le visiteur remplace l'objet dans son milieu et vit l'objet et les vies évoquées.

Cette approche affective est également favorisée par une disposition en crescendo des ceuvres. L'accrochage arrive à donner un rythme à la visite et favorise le développement d'une certaine tension dont "le point culminant sont les sinistres poupées en chiffons du dernier étage ${ }^{18}$ ".

L'intensification de l'émotion, dernière opération orientative de la lecture dont nous avons parlé, passe aussi par une utilisation des outils de création d'ambiance tels que les jeux de lumières et d'ombres; ceuxci, unis à la disposition des objets, contribuent à placer le spectateur dans un espace noir peuplé d'images qui l'enveloppent.

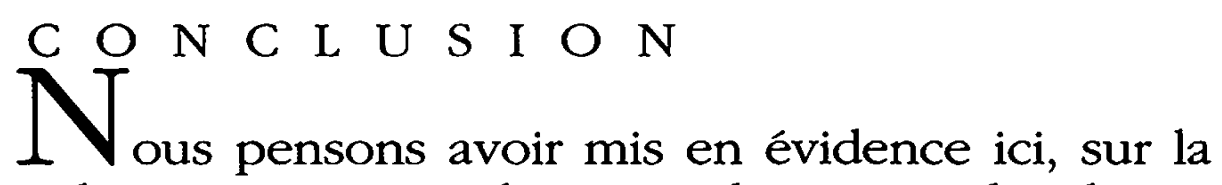
base de nos différentes observations et analyses, quelques-uns des dispositifs d'orientation de lecture de l'exposition permanente du musée de l'Art brut.

L'élaboration d'une définition du contenu du musée est un des axes sur lesquels s'articulent les discours des visiteurs. Cette définition comporte deux éléments. D'abord l'Art brut "c'est des gens", ce qu'on peut voir au musée ce sont des personnes et leur vie. Ensuite, l'Art brut, c'est des œuvres, expressions de l'intériorité de l'artiste. Il semble qu'un grand nombre de visiteurs considèrent que les objets de l'Art brut reproduisent, 
avec la fidélité d'une marine, un paysage intérieur. Les peintures sont les idées, les sentiments, l'intérieur de l'artiste, versés directement sur la toile.

Le désintérêt vis-à-vis d'une analyse formelle de l'œuvre, la préférence envers une approche émotive de l'exposition, la focalisation sur les caractéristiques psychologiques et biographiques de l'artiste, l'effort produit par le visiteur pour s'approcher de l'auteur, sont d'autres aspects du processus de lecture de l'exposition mis en ouvre par le public. Ces mouvements s'inscrivent dans une volonté générale qui transforme le musée de l'Art brut en un musée de l'Humain. Cette approche émotive par rapport à ce qui est exposé s'accorde avec une absence de considérations relatives aux concepteurs du musée ou sur le musée comme média. Comme l'affirme Morin (1978, p. 98) pour le cinéma, la qualité du spectacle-exposition est le "ressenti", l'affectivement vécu. Le visiteur du musée de l'Art brut est un visiteur désintéressé à voir l'exposition mais dédié seulement à la sentir.

Des propos des visiteurs se dégage, en conclusion, une attitude favorable à l'exposition ainsi qu'un sentiment de compréhension et de reconnaissance envers les objets montrés. Les expériences négatives par rupture du cadre attendu ne sont pas au rendez-vous. Le visiteur est possédé par un sentiment de consonance entre sa personne et ce qui est exposé, entre l'auteur et le musée, le musée et les œuvres, les œuvres et l'artiste. Les attentes et les habitudes du public qui comportaient une attente de surprise et de perturbation s'attachent sans surprise ni perturbation à ce qui leur est montré. Nous pouvons décrire la visite comme un parcours confirmatif ou chaque pas est vécu par le visiteur "... comme la réalisation d'un projet auquel, par avance, il adhère" (Heinich, 1986, p. 120).

La nature du travail de mise en scène effectué au musée de l'Art brut confère à l'offre-exposition un caractère fortement structuré. Ce travail consiste à éclairer ou à voiler des éléments des objets afin de présenter un point de vue, tout en permettant une grande variété de lectures. Les autres points de vue exposés ne sont en quelque sorte que des repoussoirs. L'exposition se développe de façon à ce que l'indétermination des objets soit levée à mesure que la visite avance et que les attentes éveillées au moment de l'entrée soient satisfaites. Nous avons parlé d'un mystère, d'une certaine question qui serait posée au visiteur, mais il nous semble évident que le visiteur n'a pas de véritable question à résoudre. La question, simple élément de rhétorique interne, ne s'ouvre pas sur un vrai mystère mais sur ce mystère de solution préexistante que nous appelons secret.

En conclusion, l'exposition apparaît comme un objet déterminé, dont les stratégies orientatives de la réception deviennent contraignantes. Le travail de guidage de la réception permet la participation du visiteur, lui donne des clés de compréhension et cependant en préparant le contenu à transmettre, réduit sa marge de spontanéité.

L'enjeu qui vise le concepteur est de taille. D'une part, il entend montrer les ouvres dans leur état naturel et éliminer tout cadre didactique afin de laisser les visiteurs libres dans leur lecture. D'autre part, il est 
responsable d'un ensemble d'œuvres dont la réunion répond à des principes théoriques qu'il s'agit de communiquer. La compréhension de ces points théoriques exige l'utilisation des dispositifs qui guident, inévitablement, la réception. Partagé entre la prétention de lisibilité au sens de rendre possible une lecture créative et l'exigence de la lisibilité au sens de favoriser l'appréhension de l'information, il nous semble qu'il gagne son pari.

E. -G. M.

Institut des sciences sociales et pédagogiques

Université de Lausanne.

Je remercie les deux lecteurs anonymes pour l'aide qu'ils m'ont apportée lors de l'élaboration de cet article.

Je tiens à exprimer ma gratitude aux professeurs Jean-Yves Pidoux et Paul Beaud de l'Institut de sociologie des communications de masse de l'Université de Lausanne qui ont dirigé cette recherche. Mes remerciements vont aussi au conservateur du musée de l'Art brut, Michel Thévoz et à ses collaborateurs qui ont facilité mon travail avec une extrême amabilité. 
1. Le musée fut inauguré le 26 février 1976. Michel Thevoz, conservateur du musée depuis 1974, prend aussi le rôle de concepteur et réalisateur de l'exposition. Compte tenu des caractéristiques du musée qui nous occupe, nous utiliserons indistinctement dans cet article les termes d'exposition et de musée.

2. Le but de notre travail consistait à montrer, par une démarche empirique, quelques modes de lecture mis en œuvre par les visiteurs de l'exposition du Musée de l'Art brut de Lausanne (Gonzalez Martinez, 1994). Nous avons envisagé l'analyse de la réception dans trois perspectives différentes. D'abord, et une fois brossée la composition socio-démographique du public et ses attentes au sujet du musée, notre attention s'est concentrée sur les démarches de lecture développées par les visiteurs en contact avec l'exposition. Ceci nous a amené à décrire cinq des figures, ou approches, que peut revêtir l'acte de lecture. Un deuxième moment de l'analyse a consisté à extraire quelques thèmes articulant les discours des visiteurs, thèmes sur lesquels se concentre leur intérêt et vis-à-vis desquels ils se positionnent diversement. Les * investissements * (Heinich \& Pollak, 1989, p. 157), dont nous avons essayé de reconstituer la logique, sont précisément les facettes de sens créées tout au long des opérations de lecture signalées antérieurement. Au travers de l'exposition, le concepteur propose au public d'accepter un " jeu * (Davallon, 1986. * Avant-propos ", p. 10) dont l'intérêt est la transmission de ses propres idées à l'égard des objets exposés. Une fois observés les différents rapports établis entre le producteur de l'exposition et le récepteur, il a été possible d'entrevoir une relation entre ces attitudes et la capacité du visiteur à "jouer le jeu de la culture * (Bourdieu, 1979, p. 365-431). La recherche de pistes explicatives des réceptions observées nous a conduit à réaliser une analyse de la structure de l'objet-exposition et à mettre en rapport la production de l'exposition et sa réception. Les matériaux empiriques utilisés pour effectuer cette recherche sont décrits dans l'introduction de cet article.

3. Nous nous sommes principalement penchés sur les propos de Dubuffet et de Thévoz (Dubuffet, 1967; Dubuffet, 1993; MacGregor, 1989; Spies, 1968; Thevoz, 1981; divers articles de presse et entretiens radiophoniques; etc.). Ce travail documentaire comporte également l'étude du projet architectural et muséologique mené à terme au musée ( Musée de l'Art Brut. Château de Beaulieun, Architecture suisse, (26), 1977, AXX10; Bulletin du Conseil Communal. Séance du mardi 10 octobre 1972. Préavis $\mathrm{n}^{\circ} 177$. Collection de l'art brut, p. 823-838; Bulletin du Conseil Communal. Séance du mardi 26 octobre 1982. Préavis $\mathrm{n}^{\circ} 215$. Château de Beaulieu, p. $1576-1582$; etc.).

4. Parmi les "témoignages de réception" du livre d'or, seuls ont été choisis les messages écrits (français, allemand, anglais, espagnol, italien et portugais) et quelques dessins se référant au musée ou à des objets s'y trouvant. Ces messages, 473 au total recouvrant la période allant d'octobre 1988 à janvier 1993, furent extraits et introduits dans une base de donnćcs afin d'en effectuer une analyse systématique. Par ailleurs, nous avons conçu des entretiens se développant dans un contexte aussi proche que possible de celui de la visite et permettant une expression verbale abondante et ouverte. Se déroulant en trois phases ces entretiens débutaient à l'entrée du musée afin de saisir les premières attentes du public au sujet de l'exposition. Une fois le visiteur à l'intérieur de l'exposition, il était invité à s'exprimer sur un choix de tableaux. A la sortie de la salle, nous avons demandé à la même personne son avis sur l'ensemble du musée. La technique utilisée lors du traitement des 
propos recueillis fut une analyse thématique des discours.

5. Le "retour aux sources - est vu par Bourdieu comme une des stratégies de subversion utilisées par les dominés pour s'imposer sur le marché : "... la stratégie par excellence est le retour aux sources qui est au principe de toutes les subversions hérétiques et de toutes les révolutions lettrées parce qu'il permet de retourner contre les dominants les armes au nom desquelles ils ont imposé leur domination..." (Bourdieu, 1977, p. 12.)

6. Toutes les déclarations entre guillemets et en italique, sauf indication contraire, sont tirées des entretiens que le conservateur du musée nous a aimablement accordés.

7. Bulletin du Conseil Communal. Séance du mardi 10 octobre 1972. Préavis $\mathrm{n}^{\circ}$. 177. Collection de l'art brut. p. 833 .

8. Préavis $n^{\circ} .177$, ibid. p. 834.

9. Notre travail s'inspire largement des thèses développées par Davallon. Les termes de "monde réel ", "monde synthétique " et " monde utopique * sont repris de son texte "Gestes de mise en exposition". (Davallon, 1986, p. 241266).

10. Pour le faire nous suivons le chemin déjà tracé par Davallon (1986), Veron (1983) ou Stichting (1989). Néanmoins, nous sommes conscientes des risques de transférer la comparaison entre la lecture et la visite d'une exposition à l'étude de la réception d'autres musées. Le comportement des visiteurs, par exemple, des éco-musées s'approche plus de la visite au luna-park ou de la réunion sociale que de la lecture individuelle et réfléchie d'une oeuvre littéraire. Voir à ce sujet l'article de O. Donnat (1993).

11. Ce travail de théorisation a été repris par Michel Thévoz, à la tête des Publications de la Collection de l'art brut et auteur d'un grand nombre d'écrits sur le musée.

12. Pour plus de renseignements concernant les trois types de lecteurs voir Jauss (1978, p. 45).
13. L'équipe de concepteurs était composée principalement par: Michel Thévoz, sa collaboratrice Geneviève Roulin, l'architecte Bernard M. Vouga et son assistant Jean de Martini.

14. L'exposition de l'énoncé emprunte, par ailleurs, un processus de construction de la réalité similaire à celui suivi pour "créer " l'art brut. Les termes de "particularisation", "généralisation", et " ouverture d'espace herméneutique* sont repris de Heinich (1991).

15. Les citations en italiques renvoient aux éléments textuels de l'exposition. Leur provenance précise est indiquée après la citation.

16. Terme repris de Davallon (1986, p. 265).

17. C'est le concept de * camouflage objectivant " développé par Greimas et utilisé par Lasalle pour décrire la rhétorique propre aux catalogues artistiques (A.-J. Greimas cité dans Lassalle, 1986, p. 406).

18. Déclaration d'un visiteur. Février 1993. Il s'agit des oeuvres de Michel Nedjar, amalgames de chiffons suspendus au plafond d'une petite chambre dans les combles du musée. 
RÉFÉRENCES BIBLIOGRAPHIQUES

Barbier-Bouvet (J.F.) et al. 1983. Histoires d'expo: un thème, un lieu, un parcours. Paris: Centre Georges Pompidou. Centre de création industrielle.

Besson (C.). 1986. "L'exposition et son lieu ", in L'objet expose le lieu. Paris: Expo-Média.

Bourdieu (P.). 1977. "La production de la croyance", Actes de la recherche en sciences sociales, (13).

Bourdieu (P.). 1979. La Distinction. Paris : Éd. de Minuit.

Bourdieu (P.). 1992. Les Règles de l'artt. Paris : Éd. du Seuil.

Carrier (C.) et al. 1986. Les Immatériaux au Centre Geonges Pompidou en 1985: étude de l'événement et de son public. Paris : Expo-Média.

Carrier (C.) et al. 1986. L'Objet expose le lieu: présentation, représentation. Paris : Expo-Média.

Clement (B.). 1983. "Le mythe de l'objet ventriloque ", in Histoires d'expo. Paris: Georges Pompidou.

Davallon (J.). 1986. Claquemurer pour ainsi dire tout l'univers. Paris: Centre Georges Pompidou.

Davallon (J.). 1992. «Le musée est-il vraiment un média?", Publics E musées, (2), p. 99-123.

De Certeau (M.). 1990. L'Invention du quotidien. Paris: Gallimard.

Desjardins (J.), Jacobi (D.). 1992. «Les étiquettes dans les musćcs ct les expositions scientifiques et techniques", Publics E musées, (1), p. 13-31.

Donnat (O.). 1993. "Les publics des musées de France ", Publics $\varepsilon$ musées, (3), p. $29-43$

Dubuffet (J.). 1967. Prospectus et tous écrits suivants. Paris: Gallimard.

Dubuffet (J.). 1968. Aspbyxiante culture. Paris : Jean-Jacques Pauvert.

Dubuffet (J.). 1993. "Art brut chez Dubuffet " (entretien avec J.-M. MacGregor), Raw Vision, (7), p. 40-51.

Gonzalez Martinez (E.). 1994. Le Visiteur à l'ouvve: analyse de la réception du musée de l'Art brut par le public. Mémoire de Maîtrise de troisième cycle en Sociologie et Anthropologie.
Université de Lausanne. Faculté des sciences sociales et politiques.

Gonzalez Martinez (E.). 1995. „La réception du musée de l'Art brut par son public: Différenciation des approches interprétatives de l'exposition*, Revue suisse de sociologie, 21(3), p. 741-761.

Heinich (N.). 1986. "Un événement culturel " in Les Immatériaux au Centre Georges Pompidou en 1985. Paris: Expo-Média.

Heinich (N.). 1987. Le Pont-Neuf de Christo: ouvrage d'art, œuvre d'art ou comment se faire une opinion. Paris: Adresse.

Heinich (N.). 1989. "Au pied du mur: sur une fresque en milieu urbain ", Sociologie et Sociétés, XXI(2), p. 91101.

Heinich (N.). 1991. La Gloire de Van Gogh. Paris: Éd. de Minuit.

Heinich (N.), Pollak (M.). 1989. *Du conservateur du musée à l'auteur de l'exposition: l'invention d'une position singulière*, Sociologie du travail, 31(1), p. 29-49.

Heinich (N.), Pollak (M.). 1989. Vienne à Paris: portrait d'une exposition. Paris: Centre Georges Pompidou. Bibliothèque publique d'information.

Iser (W.). 1985. L'Acte de lecture. Bruxelles: Mardaga.

Jauss (H.-R.). 1978. Pour une esthétique de la réception. Paris: Gallimard.

Lassalle (H.). 1986. "Les exclusives du discours sur l'art ", Sociologie de l'art. Paris : La Documentation française.

MacGregor (J.-M.). 1989. The Discovery of the Art of the Insane. Priceton: Priceton University.

Morin (E.). 1978. Le Cinéma ou l'bomme imaginaire: essai d'anthropologie sociologique. Paris: Minuit.

Passeron (J.C.), Pedler (E.). 1991. Le Temps donné aux tableaux: compte rendu d'une enquête au musée Granet. Marseille : Cercom/IMEREC.

Perraton (C.) 1987. "Voir et toucher la science" p. 15-66, in Schiele et al., Ciel, une expo! Paris : Expo-Média.

Poli (M.-S.). 1992. «Le parti pris des mots dans l'étiquette: une approche linguistique ", Publics E musées, (1), p. 91-103. 
Rutten.(F.). 1980. « Sur les notions de texte et de lecture dans une théorie de la réception", Revue des sciences bumaines, 59(177), p. 72.

Schiele (B.) et al. 1987. Ciel, une expo!: approche de l'exposition scientifique. Paris : Expo-Média.

Schiele (B.). 1992. "L'invention simultanée du visiteur et de l'exposition ", Publics E musées, (2), p. 71-97.

Soisson (J.). 1973. "Aux alentours de l'Art brut ", in Dubuffet. Paris : L'Herne.

Solomon (R.). 1973. Jean Dubuffet. New York: The Solomon R. Guggenheim Foundation.

Spies.(W.). 1968. "L'Art brut avant 1967", Revue de l'art, (1-2), p. 123-126.

Stichting (C.). 1989. La Présentation muséale comme narration. Amsterdam: Camini Stichting.

Thevoz (M.). 1974. in Cultiver l'Art brut/sous la direction de A. Willener \& $\mathrm{T}$. Burnier. Lausanne: Institut de recherches des communications de masse.

Thevoz (M.). 1981. L'Art brut. Genève: Skira.

Thevoz (M.). 1985. "Une heure avec Michel Thévoz*(entretien), Nouvelle revue de Lausanne, 21 octobre 1985.

Thevoz (M.). 1986. Dubuffet. Genève: Skira.

Thevoz (M.). 1989. in Une approche des musées/sous la direction de A. Mottaz $\& \mathrm{P}$. Christiane. Lausanne: Institut d'anthropologie et sociologie.

Vergo (P.) et al. 1989. The New Museology. London: Reaktion Books.

Veron (E.), Levasseur (M.). 1983. Ethnographie de l'exposition: l'espace, le corps et le sens. Paris: Centre Georges Pompidou. Bibliothèque publique d'information

Veron (E.). 1986. Les Spectacles scientifiques télévisés: figure de la production et de la réception. Paris : La Documentation française

Veron (E.). 1992. "Le plus vieux média du monde ", Mediascope, (3), p. 32-37. 
L'article présente une analyse de l'exposition permanente du musée de l'Art brut de Lausanne. L'exposition est vue comme un artefact communicatif capable de prendre de multiples significations en fonction des réceptions dont il fait l'objet. Néanmoins, cet artefact, pour être constitué et compris, formule des directives à suivre. L'auteur, devenu visiteur averti, tente de dégager la structure de l'exposition par l'interprétation de données faisant référence autant à sa production qu'à sa réception. Il met ainsi en évidence cinq opérations orientatives de la réception considérées comme autant de moments d'une stratégie communicationnelle susceptible d'être à l'origine des réceptions observées.

\section{$T_{\text {bis article presents our analysis of the perma- }}$} nent exbibition at the Raw Art Museum (musée de l'Art Brut) in Lausanne, Switzerland. This exhibition can be viewed as an communicational artefact that takes on multiple meanings according to the various ways it is received by visitors. Nevertheless, in order for it to be constituted and understood, this artefact should include a series of operating instructions. The author, an informed visitor, tried to bring out its structure by interpreting data on both production and reception of the exbibition. She underscores five directional operations of reception; these may be regarded as a so many phases of a communications strategy that could be one of the foundations of vistors' interpretations.

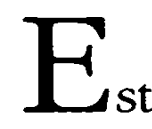

ste artículo presenta un análisis de la exposición permanente del Museo de Arte Bruto (musée de l'Art Brut) de Lausana, Suiza. La exposición es considerada como un artefacto comunicativo capaz de adoptar distintos significados según las recepciones de las cuales es objeto. Este artefacto incluye instrucciones para ser recreado por el publico. La autora saca a la luz la estrutura de la exposición mediante la interpretación de datos que hacen referencia tanto a la producción como a la recepción del museo. Se muestran cinco operaciones orientativas de la recepción consideradas como otros tantos momentos de una estrategia comunicativa susceptible de estar al origen de las recepciones observadas. 